\title{
VACío CULTURAL Y AUTENTICIDAD: CARLA LONZI
}

\section{Cultural vacuum and authenticity: Carla Lonzi}

\author{
Gemma del Olmo Campillo \\ gdelolmo@unizar.es \\ Universidad de Zaragoza-España
}

Recibido: 25-02-2017

Aceptado: 23-05-2017

\section{Resumen:}

La crítica a la cultura occidental realizada por el movimiento feminista es relevante tanto para poner en evidencia que Occidente procede de forma colonialista con los sujetos y culturas que considera inferiores, como para llevar a cabo el necesario trabajo de autocrítica dentro de los feminismos occidentales que tienen que asumir sus prejuicios con respecto a subjetividades y culturas marginadas, y por tanto hacer las transformaciones precisas en sus perspectivas y análisis para acabar con las tendencias colonialistas. La reflexión se centra en Carla Lonzi por ser una autora que en todas sus obras mantiene la necesidad de realizar una crítica radical a la cultura.

Palabras clave: Carla Lonzi, autenticidad, reconocimiento, desculturación, cultura.

\begin{abstract}
:
The critique of Western culture carried out by the feminist movement is significant because it makes clear that the West adopts a colonialist approach to subjects and cultures it considers inferior. It is necessary for the work of self-criticism within Western feminisms that they recognize their prejudices regarding subjectivities and marginalized cultures, allowing necessary transformations to end colonialist tendencies. The reflection focuses on Carla Lonzi for being an author who in all her works maintains the need to make a radical critique of culture.
\end{abstract}

Keywords: Carla Lonzi, authenticity, recognition, deculturation, culture. 


\section{Introducción}

Puede considerarse que los feminismos más actuales suponen una ruptura con respecto a las perspectivas feministas anteriores, en cuestiones como por ejemplo la prostitución, la pornografía o las sexualidades disidentes, sin embargo, no es menos cierto que, para poder ofrecer sus nuevos planteamientos, dichos análisis se apoyaron en muchas de las reflexiones previas, lo que en cierta medida supone un cuestionamiento de las interpretaciones que consideran los nuevos feminismos una fractura con las autoras precedentes y los feminismos clásicos. Poniendo solo algún ejemplo, autoras como Adrienne Rich, Carla Lonzi, Kate Millett, Monique Wittig, Anne Koedt o Gayle S. Rubin insistieron en subrayar la relevancia del placer y la sexualidad femenina en sus análisis sobre la libertad de las mujeres, lo que posibilitó nuevos enfoques que fueron desarrollados después, aunque de una forma distinta.

Pero la sexualidad no es el único aspecto relevante abierto por los feminismos anteriores a la década de los 90 del siglo XX, quisiera destacar otro, que para estas autoras está relacionado con la sexualidad, me refiero a la crítica a la cultura occidental. Me gustaría centrarme en esto último porque, en mi opinión, fue crucial en los análisis sobre los prejuicios raciales, de clase y heteronormativos, entre otros, característicos de una cultura que se había erigido a sí misma modelo de convivencia, progreso, libertad y justicia.

A partir de las críticas realizadas por estos feminismos, la cultura occidental se reveló misógina y jerárquica, defensora de los intereses de un grupo claramente privilegiado: el de los hombres occidentales blancos, propietarios y heterosexuales. Dicha estructura se mantiene en la medida en que pueda preservar esos privilegios, ya sea a través de ejercicios de poder o a través de argumentaciones que intenten justificar la jerarquía y privilegios existentes. Las explicaciones más conocidas han sido las que han recurrido a una naturaleza superior de los hombres, así como las que insisten en conservar la situación tal y como está en aras de las tradiciones, del beneficio de una convivencia con roles ya establecidos, etc.

Hay, en este sentido, una convergencia de objetivos con aquellos movimientos sociales que se caractericen por tener posiciones críticas respecto a los privilegios de los sujetos favorecidos que delimitan el concepto de "normalidad". Esta confluencia ha sido subrayada por los feminismos que consideran que las exclusiones sociales están interrelacionadas porque proceden, en general, de una lógica de discriminación ejercida por un sistema que maltrata cualquier disidencia.

Además, siguiendo esta línea crítica, el movimiento feminista realizó un ejercicio de reflexión sobre sí mismo al poner en evidencia los propios prejuicios hacia otras culturas y, con ello, la necesidad de un ejercicio de autocrítica que reconociera las limitaciones y arbitrariedades de los feminismos occidentales hacia los otros feminismos, así como el compromiso de trabajar siendo muy conscientes de las perspectivas desde las que se hablaba para, de esta forma, paliar o 
eliminar las exclusiones de otras subjetividades o culturas marginadas. Esta autocrítica es hoy ineludible para los feminismos que reivindican la diversidad y que no están dispuestos a repetir los dañinos ejercicios de exclusión ya realizados por las sociedades, sobre todo la occidental.

Así, se puede afirmar que la crítica a la cultura occidental, llevada a cabo por los feminismos anteriores a los años 90 del siglo XX, se vio enriquecida por planteamientos posteriores que aportaron nuevos e importantes elementos de autocrítica que señalaron las omisiones en las que había incurrido el movimiento feminista, sin que ello tenga que significar necesariamente una ruptura ni un menosprecio hacia los esfuerzos y las aportaciones de los feminismos previos.

\section{Crítica radical a la cultura}

Con la idea de subrayar las posibilidades de análisis que abrieron los feminismos de los años 70 y 80, me gustaría centrarme en una autora principalmente: Carla Lonzi, porque su pensamiento se caracteriza, precisamente, por su crítica a la cultura occidental. Es cierto que hay muchas otras que tienen afirmaciones en este sentido, pero quizá Lonzi es una de las que más hincapié hace durante toda su producción. Encontramos ya las siguientes palabras en una de sus primeras obras, en el prólogo de su famoso Escupamos sobre Hegel: "Al tomar conciencia de los condicionamientos culturales que desconocemos, no dudamos siquiera de que habíamos descubierto algo esencial, algo que cambiaba todo: el sentido que tenemos de nosotras, de las relaciones, de la vida" (Lonzi, 1975: 10).

Es decir, Lonzi señala a la cultura y sus condicionamientos como un espacio fundamental de opresión, por lo que darse cuenta de ese papel opresivo que tiene en la vida de todas las mujeres es el inicio necesario para una transformación profunda de la sociedad. En la misma obra de Lonzi, se publicó el primer manifiesto del grupo Rivolta Femminile (grupo que fundó Lonzi junto a Carla Accardi), texto del que Lonzi se siente personalmente responsable, aunque esté firmado por el grupo (Boccia, 1990: 106). En este manifiesto fundacional se hace la siguiente afirmación: "la fuerza del varón reside en su identificación con la cultura, la nuestra en su refutación" (Rivolta Femminile, 1975: 19). De modo que la fuerza del movimiento feminista está en la crítica y rechazo de la cultura patriarcal por ser una ideología que responde a unos intereses en detrimento de otros, y dado que los intereses que prevalecen son los de las personas y cuerpos privilegiados, el grupo de Carla Lonzi hace la siguiente denuncia: "detrás de toda ideología adivinamos la jerarquía de los sexos" (Rivolta Femmile, 1975: 17).

No hay ninguna producción cultural occidental, ni el arte, ni la política de partidos, ni las religiones, ni la filosofía, por poner algunos ejemplos, que esté a salvo de esta estructura en la que hay un menosprecio de las mujeres y de los aspectos asignados a lo femenino. Toda creación cultural lleva consigo esta servidumbre, por lo que es absolutamente necesaria no solo una crítica 
a la cultura occidental, sino la creación de otra cultura para que sea posible la libertad de las mujeres. Propone una transformación radical: la erradicación de una cultura narcisista, orgullosa de sí, que se considera superior a otras culturas pero que en realidad muestra muy poca capacidad para la autocrítica y aprecio de la diversidad. Es cierto que ha habido muchos desarrollos técnicos y grandes pensadores, pero estas aportaciones y reflexiones beneficiaban sobre todo a una parte de la humanidad:

"Consideramos responsables de las grandes humillaciones que nos ha impuesto el mundo patriarcal a los pensadores: ellos son quienes han mantenido el principio de la mujer como ser adicional para la reproducción de la humanidad, vínculo con la divinidad o umbral del mundo animal; esfera privada y pietas. Ellos han justificado en la metafísica lo que en la vida de la mujer había de injusto y atroz" (Rivolta Femminile, 1975: 19).

También en las últimas publicaciones de Lonzi se puede apreciar que mantiene su posición crítica respecto a la cultura, y sigue considerando importante acabar con ella para conseguir la libertad de las mujeres. Así, en su texto titulado "Itinerario de reflexiones", publicado en 1977, propone un vacío cultural con el que identificarse (Lonzi, 2012: 78), un vacío desde el que construir el sentido propio y una nueva forma de convivencia social, más justa, libre y auténtica. Dicha propuesta de Lonzi es similar a la de sus primeros textos, lo que va variando es la forma de expresar la misma idea. Por ejemplo, en su primera aparición, en Escupamos sobre Hegel, este vacío cultural se presenta con el término de "desculturación" (Lonzi 1975: 45), mientras que en otra, Taci, anzi parla, utiliza la expresión “tabula rasa” (Lonzi, 2010: 53).

"Nuestra acción es la desculturación por la que optamos. No se trata de una revolución cultural que sigue e integra la revolución estructural, no se basa en la verificación a todos los niveles de una ideología, sino en la carencia de necesidad ideológica. La mujer no ha contrapuesto a las construcciones del varón más que su dimensión existencial: no han salido de entre ellas jefes, pensadores, científicos, pero ha poseído energía, pensamiento, coraje, decisión, atención, sentido, locura. Las huellas de todo esto se han borrado porque no estaban destinadas a perdurar, pero nuestra fuerza estriba en no poseer ninguna mistificación de los hechos: actuar no es una especialización de casta, aunque se convierte en ello mediante el poder por el que está orientada la acción. La humanidad masculina se ha adueñado de este mecanismo cuya justificación ha sido la cultura. Desmentir la cultura significa desmentir la valoración de los hechos que constituyen la base del poder" (Lonzi 1975: 45 46).

La propuesta de "desculturación”, de vacío cultural, que hace Lonzi parece difícil de realizar, pero a ella le parecía factible, y no solo eso, consideraba que llevarla a cabo era algo absolutamente necesario. Para lograrlo confiaba en las posibilidades de transformación personal y social de los grupos de autoconciencia, tan importantes en el feminismo en los últimos años de 
la década de los 60 y los primeros de los 70 del siglo XX. Estos grupos no eran, en su opinión, la única manera, pero sí la más efectiva. En ellos se escuchaba la voz y los deseos de muchas mujeres y, a partir de ellos, los propios. El inicio de la libertad, para Lonzi, es nombrar, pensar y reflexionar sobre las propias experiencias, sin utilizar los instrumentos teóricos construidos por los hombres detrás de los cuales siempre subyace el menosprecio de lo que no es el sujeto privilegiado. Es el comienzo de la búsqueda de autonomía de juicio, con la ayuda de otras mujeres feministas, pero dejando fuera el deseo de la aprobación masculina, que en realidad nunca llegaba porque, en su opinión, las mujeres no forman parte de lo que ellos valoran.

Advierte que el engaño de la cultura ha sido muy claro: a las mujeres se les ha prometido el reconocimiento de su subjetividad si obedecían, si acataban las normas culturales que, en realidad, desprecian a las mujeres y lo femenino. Un círculo viciado y vicioso del que no se puede salir: las mujeres obtienen un cierto reconocimiento cuando estas aceptan su papel subordinado. Así las cosas, las mujeres no pueden ser libres en la cultura occidental, tal y como esta se ha venido desarrollando. Ni las mujeres ni otras culturas ni las identidades excluidas, se podría añadir.

El vacío cultural que propone Lonzi, no es, por tanto, volver a un estado de naturaleza, algo que a todas luces es imposible, sino salir de la cultura tan jerárquica que caracteriza a Occidente y construir una nueva que no desprecie a otras sociedades, en la que se pueda convivir en igualdad de condiciones, con iguales oportunidades, sin menosprecios ni jerarquías. Quiere una forma distinta de relacionarse, en la que no se instrumentalice a las otras personas, ni se manipule, ni se huya de los problemas. Quiere tener lo que ella llama relaciones auténticas, que se pueden entender como relaciones basadas en la confianza, la vulnerabilidad y el reconocimiento.

Esta manera de relacionarse es la que considera que se puede desarrollar en los grupos de autoconciencia, no solo, claro, pero sí confía en estos espacios como lugares fundamentales para impulsar nuevas formas de valorar y de relacionarse, lo cual no significa que deje de reconocer la existencia de problemas de relación entre mujeres. Lonzi critica abiertamente la mitificación de los grupos, porque es consciente de que en ellos surgen también fuertes desavenencias. La disparidad a veces es difícil de superar, pero es un esfuerzo ineludible si lo que se pretende es llegar al descubrimiento de la propia autenticidad, si lo que se quiere es llegar a la manifestación de sí. Para iniciar el camino hacia ese desvelamiento es preciso situarse fuera de los sistemas jerárquicos que inculcan prejuicios tendenciosos contra las mujeres y lo femenino, y que, además, establecen maneras de relacionarse instrumentales porque manipulan a otras personas en su propio beneficio.

La propuesta de Lonzi conlleva un esfuerzo personal grande, como se puede apreciar en su obra Taci, anzi parla, porque lo que pretende Lonzi no es solo criticar las ideas con las que se ha crecido, ideas que se han considerado propias, que ya es un trabajo arduo, sino que también supone criticar convicciones de las personas con las que se tiene una relación cotidiana. Lonzi 
está pidiendo una crítica profunda a la cultura que, en sentido estricto, implica además de una autocrítica, un cuestionamiento de las personas con las que se convive estrechamente.

Piensa que esta (auto)crítica es un paso imprescindible para transformar y mejorar la vida de todas las personas que conviven en una sociedad, porque para Lonzi el feminismo es un pensamiento que quiere mejorar la vida y la convivencia. Ese es el sentido de su trabajo; no es algo meramente teórico. "El feminismo no solo es rabia, denuncia, sino también autoconsciencia y liberación, es todo el arco, todas las fases de un proceso: el resultado es siempre el descubrimiento de sí. El estar en un proceso, en lugar de otra cosa, modifica la civilización" (Lonzi, 2010: $161^{1}$ ).

Los grupos de autoconciencia posibilitan la reflexión que se precisa para tomar conciencia del daño causado por la cultura, pero el trabajo de re-construcción es una labor que requiere mucho esfuerzo personal y autenticidad. El grupo puede mostrar lo dañinas que han sido las expectativas sociales y los roles asignados para la expresión de sí, pero esto sirve de poco si no se modifica el propio comportamiento. Cada cual tiene que responsabilizarse de lo que hace y de lo que no hace, arriesgar en su vida personal si lo que de verdad quiere es apostar por una cultura que no instrumentalice a las personas. Para Lonzi es posible re-construirse de forma voluntaria y reflexiva, con el propósito de convertir a la sociedad en un lugar más habitable para todo el mundo, aunque al principio esto pueda poner en riesgo las relaciones con las personas más cercanas.

La autenticidad es otro de los elementos interesantes que Lonzi mantiene en bastantes obras, aparece en uno de los primeros textos firmados por Rivolta Femminile (del que Lonzi también se siente personalmente responsable), con la afirmación: "El feminismo comienza cuando una mujer busca la resonancia de sí en la autenticidad de otra mujer" (Rivolta Femminile, 1975: 126). Si bien es cierto que en ocasiones tenía dudas sobre su apuesta por la autenticidad (Lonzi, 2010: 821), también lo es que es una idea muy presente en sus textos, y de forma contundente. Por ejemplo, en el primer manifiesto de Rivolta Femminile encontramos la siguiente afirmación: "Nosotras buscamos la autenticidad de gesto de rebelión y no la sacrificaremos ni a la organización ni al proselitismo" (Rivolta Femminile, 1975: 20). Y también en su diario: "Si pierdo de vista la autenticidad ya no sé quién soy." (Lonzi, 2010: 636 ${ }^{2}$ ).

Para Lonzi la liberación solo es posible en la autenticidad (Lonzi, 2010: 132); es un elemento principal para el cambio y la transformación social. Solo teniendo relaciones auténticas y no instrumentales, sin mentiras ni manipulaciones, será posible una nueva cultura. Además, esa autenticidad conlleva otro de los elementos básicos del pensamiento de Lonzi: el reconocimiento. El reconocimiento es indispensable para poder salir de la situación de ocultamiento a la que se

\footnotetext{
${ }^{1}$ Traducción de la autora.

2 Traducción de la autora.
} 
ven abocadas las mujeres en la cultura occidental, pero solo un proceder desinteresado hará posible que se reconozca la valía de las mujeres, sin manipulaciones ni falsedades.

Hay que aclarar que el planteamiento de Lonzi está lejos de la conocida propuesta de Hegel expresada en su Fenomenología del Espíritu, en la dialéctica del amo y del esclavo, donde es necesaria la hostilidad entre las conciencias, la lucha, para llegar al reconocimiento: "Por consiguiente, el comportamiento de las dos autoconciencias se halla determinado de tal modo que se comprueban por sí mismas y la una a la otra mediante la lucha a vida o muerte. Y deben entablar esta lucha, pues deben elevar la certeza de sí misma de ser para sí a la verdad en la otra y en ella misma" (Hegel, 1978: 116). El reconocimiento al que se refiere Lonzi está basado en la autenticidad, una relación sin luchas y sin instrumentalizaciones, sin duelos ni rivalidades (Lonzi, 2010: 807). El reconocimiento sería, entonces, junto con la autenticidad, un pilar básico sobre el que asentar la nueva cultura propuesta por Lonzi.

En esta nueva forma de convivencia sí se reconocería a los sujetos que sistemáticamente no lo son en la cultura misógina. De ahí que en el prólogo de su obra Vai pure escriba lo siguiente: "Vai pure es la grabación en cuatro jornadas del momento de recapitulación de una relación en los puntos inconciliables de dos individuos que son dos culturas: la de la mujer que trata de poner las bases para su reconocimiento, y la del hombre que se refiere a la necesidad de 'lo que es', que son sus necesidades" (Lonzi, 2011: $3^{3}$ ).

El reconocimiento es un aspecto fundamental, vital, del ser humano, no es una cuestión superficial de orgullo o de cortesía, es una cuestión que afecta no solo a la imagen que una persona tiene de sí, sino también al sentido que se da a sí y a lo que le rodea. Afecta, pues, a elementos tan básicos como el hecho de significar y significarse. Y su escasez o carencia resulta demoledora: "la falta de repercusión produce sobre quien la sufre el efecto de no existir, de ser un error viviente" (Lonzi, 1978: 148).

En la cultura occidental, como también ha sido reflejado por Hegel, hay sujetos para los que se abre la posibilidad de significarse y ser reconocidos, y otros que no tienen esa opción. Pues bien, Lonzi apuesta por el reconocimiento de esos otros sujetos insignificantes que quieren vivir en libertad y significar. Apuesta por las mujeres y por el reconocimiento de unas a otras para romper con la dinámica de que solo los hombres sean reconocidos. Así, afirma "si no nos reconocemos la una a la otra quien es reconocido es el hombre: de esta manera se difunde su cultura" (Lonzi, 1978: 137).

Este análisis puede servir también para otros sujetos en la misma situación de falta de reconocimiento social, porque la cultura ha utilizado instrumentos similares en sus exclusiones:

"En resumen, la cultura masculina opera en sentido colonial, por debajo de la cultura: decide cuál es el feminismo a declarar como tal, omite el resto, reconoce como válida cada manifestación ambigua

\footnotetext{
${ }^{3}$ Traducción de la autora.
} 
de mujeres en la que esté presente la aspiración cultural, da permisos revolucionarios a las que aceptan ser escritoras, pintoras, artistas, comediantes, políticas, con eso mismo pone a salvo sus valores que son jerárquicos y categoriales. Todo lo que parece existencialmente sin identidad atribuible al ejercicio de un papel social, lo suprime. Y así suprime a las mujeres, y a su conciencia, de lo que es auténtico." (Lonzi, 2010: 9504).

Con estas palabras Lonzi aborda la problemática de cómo la cultura absorbe y neutraliza los movimientos contraculturales, y las reivindicaciones de los sujetos no reconocidos por la sociedad. La cultura solo parece aceptar dar entidad a aquellos sujetos o movimientos que se sometan, en mayor o menor medida, a la ideología y al orden preestablecidos, fuera de eso no hay reconocimiento sino ocultamiento y colonización. Precisamente este ejercicio constante de colonización es una característica de la cultura occidental y la forma mediante la que se relaciona con todo sujeto no hegemónico.

A través de distintos recursos, la cultura ofrece promesas de reconocimiento a los sujetos excluidos, pero no se trata de un reconocimiento real, sino de una incierta aceptación siempre y cuando se asuma su lugar en los márgenes. Como en el caso de algunas mujeres artistas que señala Lonzi (2010: 502), donde la cultura elimina sus elementos de autenticidad y acoge sus propuestas desde una posición de tolerancia porque son recibidos como si fueran una excepción a la regla: mujeres excepcionales cuyo genio único en principio no modifica la poca estimación hacia el resto de mujeres.

\section{Placer y autenticidad}

La pretensión de Lonzi es impulsar un cambio radical que acabe con esta situación. Propone, como ya se ha resaltado, la "desculturación" para crear una cultura auténtica y no instrumental, una cultura en la que la libertad de las mujeres sea posible. Y, posiblemente, la de otras diferencias también.

En este punto me gustaría hacer alguna concesión al tiempo actual y señalar que en cierto modo esto ha ido ocurriendo ya, el reconocimiento de las diferencias parece cada vez mayor, somos cada vez más conscientes de los ejercicios de colonización realizados por la cultura, así como de los daños ocasionados. Se han realizado importantes avances en este sentido desde los años 70 hasta la actualidad, aunque no puedo dejar de advertir que desde luego no son en modo alguno suficientes, aún queda mucho por hacer.

\footnotetext{
${ }^{4}$ Traducción de la autora.
} 
Por otra parte, también quisiera puntualizar que no ignoro el hecho de que hablar del reconocimiento de las diferencias, en plural, sin atenerme únicamente a la diferencia sexual, quizá pueda parecer una aspiración un tanto alejada de las propuestas de Carla Lonzi y de su grupo Rivolta Femminile, sobre todo si tenemos en cuenta sus ambigüedades respecto a una de estas diferencias: el lesbianismo. Las vacilaciones a las que me refiero son las que tienen que ver con afirmaciones que parecen sugerir que toda sexualidad libre es política, como: "aceptamos la libre sexualidad en todas sus formas" (Rivolta Femminile, 1975: 18), que se ven oscurecidas por otras en las que aseguran que el lesbianismo no es una alternativa política (Rivolta Femminile, 1978: 176).

La confusión es aún mayor en algunos párrafos del diario de Lonzi. Sin embargo, con todo, la relevancia que tiene para esta autora la sexualidad es tal que no me parece demasiada temeridad abrir su análisis a las sexualidades no convencionales, y su propuesta de vacío cultural al reconocimiento de otras diferencias. Para ello, quisiera referirme a su conocido texto "La mujer clitórica y la mujer vaginal” (Lonzi, 1975: 67-120). En él, Lonzi vincula unas prácticas sexuales con la aceptación de la cultura patriarcal y otras con su rechazo y resistencia, esto es, hay una relación clara entre la sexualidad clitórica y las mujeres que no renuncian al propio placer ni se someten al placer de los hombres: "La mujer vaginal es aquella que, en cautiverio, ha sido llevada a una actitud consentidora para goce del patriarca: mientras que la mujer clitórica es la que no ha condescendido a las sugestiones emotivas de integración con el otro" (Lonzi, 1975: 73). Establece, pues, una clara conexión entre la valoración el propio placer y no depender psicológicamente del reconocimiento del hombre:

"Para gozar plenamente del orgasmo clitórico la mujer debe alcanzar una autonomía síquica respecto del varón. Esta autonomía síquica es tan inconcebible para la cultura masculina que es interpretada como rechazo del varón, como presupuesto de una inclinación hacia las mujeres. Por eso el mundo patriarcal le reserva, además, el ostracismo con el que se condena todo aquello que se sospecha como apertura a la homosexualidad" (Lonzi, 1975: 72-73).

El placer femenino estaría localizado para Lonzi en el clítoris: "El sexo femenino es el clítoris, el sexo masculino es el pene" (Lonzi, 1975: 69). No voy a entrar en las complejidades y dificultades de esta afirmación, porque considero relevante no tanto su adecuación con la realidad cuanto la fuerza transformadora o conservadora que esta autora confiere a la sexualidad. Así, en su planteamiento, la reivindicación del placer sexual femenino supone un posicionamiento revolucionario y de resistencia. Las mujeres clitóricas podrían considerarse, así, los sujetos revolucionarios de la propuesta de Lonzi, por la relación directa que establece entre la sexualidad no convencional y la transformación social.

Sin embargo, matizando la afirmación anterior, Lonzi no hace la misma afirmación respecto al lesbianismo, aunque ella considere que la sexualidad clitórica forma parte del lesbianismo: 
"Nosotras queremos afirmar el amor clitórico como modelo de sexualidad femenina en la relación heterosexual, pues no nos basta tener el clítoris como punto de referencia consciente durante el coito, ni queremos que la oficialidad del clítoris pertenezca a la relación lesbiana" (Lonzi, 1975: 102). Esto es, considera revolucionaria una sexualidad que no esté sometida al placer del hombre, pero quizá dentro de una relación heterosexual, más en la línea de Beauvoir que, desde luego, en la de Wittig:

"El hombre se siente más molesto ante una heterosexual activa y autónoma que ante una homosexual no agresiva; la primera cuestiona las prerrogativas masculinas; los amores sáficos están lejos de contrariar la forma tradicional de división de los sexos: en la mayoría de los casos son una forma de asumir la feminidad, no de rechazarla" (Beauvoir, 2011: 520).

"Las mujeres sólo pueden entrar en el contrato social (es decir, uno nuevo) escapando de su clase, incluso si tienen que hacerlo como esclavas fugitivas, una por una. Ya lo estamos haciendo. Las lesbianas somos desertoras, esclavas fugitivas" (Wittig, 2010: 71).

Para Lonzi, el lesbianismo (y se podrían añadir otras formas no normativas de sexualidad) es una opción personal de cada cual, y la lucha política para cambiar radicalmente la cultura estaría más en la dirección de apostar por una nueva forma de relacionarse basada en la autenticidad que en los cambios que se pueden ocasionar con sexualidades alternativas. Lo cual no es del todo coherente, como he señalado ya, con otras afirmaciones, en especial con las relativas a las mujeres clitóricas. Es decir, si las mujeres clitóricas son las que se resisten a la colonización sexual y no renuncian a su placer por el de los hombres, y son las que pueden llevar a cabo el vacío cultural, entonces las lesbianas estarían incluidas entre ellas, y de manera especial, según la afirmación de Lonzi (citada unas líneas antes) en la que manifiesta no querer dejar "la oficialidad del clítoris" a las lesbianas.

Sin querer profundizar más en esta problemática, lo que parece claro es que su propuesta está en la óptica de persistir en la idea de que las mujeres no pueden renunciar al placer sexual, no deben aceptar que su placer sea subsidiario, sino que tienen que situarlo en el lugar central que merece, ya sea en prácticas heterosexuales o en las homosexuales.

"La mujer clitórica representa todo lo auténtico e inauténtico del mundo femenino que ha logrado separarse del visceralismo con el varón. Auténticamente, por cuanto ésta se ha reivindicado a sí misma; enajenándose la otra [la mujer vaginal] porque ha simulado en el terreno del placer, ha codiciado el nivel del varón en el terreno cultural y social" (Lonzi, 1975: 74).

Las vidas no convencionales tienen la capacidad de poner en cuestión la universalidad o idoneidad de las ideas en las que se apoyan las propuestas más aceptadas socialmente. Aunque 
no pretendan hacerlo, el mero hecho de vivir de otra forma, a pesar de las dificultades, hace que se ponga en cuestión lo que se ha prescrito como únicas formas aceptables de vida.

La sexualidad se incorpora con fuerza al pensamiento crítico porque es uno de los ámbitos sobre los que la sociedad ha ejercido y ejerce mayor control. Por este motivo, Lonzi considera que ser conscientes de los ejercicios de colonización realizados por la cultura sobre nuestras prácticas sexuales es uno de los inicios del camino de la liberación. A pesar de que sus análisis no incluyen a las sexualidades disidentes, al menos no de una forma explícita, sí considera que la sexualidad es un espacio tan relevante que puede ser el lugar de partida para la liberación de las mujeres. "La primera condición de despegue en la existencia femenina es reconocer en la colonización sexual la condición básica del debilitamiento y del sometimiento de la mujer. Es ahí de donde toda mujer debe partir para liberarse" (Lonzi, 1975: 94).

\section{Conclusiones}

El placer sexual es, para algunas feministas de los años 70, entre las que se encuentra Lonzi, un espacio destacado de transformación personal que, a su vez, conlleva la demanda de más cambios: "Millones de mujeres que desde hace tanto tiempo expresan un malestar profundo y universal por el sexo son una constante en la historia de la humanidad femenina que denuncia y reafirma la necesidad de una transformación del mundo" (Lonzi, 1975: 77).

La crítica a la cultura y los análisis sobre el placer femenino realizados en los años 70 y 80 cuestionaron el modelo hegemónico de convivencia en Occidente, mostraron la capacidad que tenía el sistema establecido para someter, su habilidad para el control de la vida privada, de la cotidianeidad, del placer sexual... y sus consecuencias en la psique de las mujeres y en las subjetividades no privilegiadas.

Las feministas de estos años, entre las que estaba Lonzi, creían que tomar conciencia de la situación de exclusión de las mujeres, de la existencia de un grupo favorecido que no quiere renunciar a sus ventajas, podía ser el inicio del fin. El final de una cultura en la que la vida de las mujeres se desarrolla "al límite de lo invivible" (Lonzi, 2011: 30), y el comienzo de una nueva forma de relacionarse, en la que las diferencias fueran consideradas una riqueza y fueran conceptualizadas lejos de cualquier ejercicio de subordinación. Este cambio, para Lonzi, requeriría el vacío cultural que ella formula, a partir del cual serían posibles vínculos auténticos en libertad. 


\section{BIBLIOGRAFÍA}

- Beauvoir, Simone de (2011): El segundo sexo. Madrid: Cátedra.

- Boccia, Maria Luisa (1990): L'io in rivolta. Vissuto e pensiero di Carla Lonzi. Milán: La Tartaruga.

- Hegel, Georg W. F. (1978): Fenomenología del Espíritu. México: Fondo de Cultura Económica.

- Lonzi, Carla (1975): Escupamos sobre Hegel. Y otros escritos de Liberación femenina. Buenos Aires: La Pleyade.

(1978): "Mito della proposta culturale”. En: Marta Lonzi, Anna Jaquinta, Moderata Fonte

y Carla Lonzi: La presenza dell'uomo nel femminismo. Milán: Scritti di Rivolta Femminile, $\mathrm{n}^{\circ}$. 9, pp. 137-154. Disponible en: http://www.ub.edu/duoda/bvid/text.php?doc=Duoda:text:2016.07.0001 [18/02/2017]. . (2010): Taci, anzi parla. Diario di una femminista. Vol. I (1972-1973) y Vol. II (19741977). Milán: Et al. Edizioni. . (2011): Vai pure. Dialogo con Pietro Consagra. Milán: Et al. Edizioni. . (2012): "Itinerario de reflexiones". En: Duoda. Estudios de la diferencia sexual, nº. 42, pp. 56-91.

- Lonzi, Marta; Jaquinta, Anna; Fonte, Moderata y Lonzi, Carla (1978): La presenza dell'uomo nel femminismo. Milán: Scritti di Rivolta Femminile.

- Millett, Kate (2010): Política sexual. Madrid: Cátedra.

- Rivolta Femminile (1975): “Manifiesto". En: Carla Lonzi: Escupamos sobre Hegel. Y otros escritos de Liberación femenina. Buenos Aires: La Pleyade, pp. 13-20.

. (1975): "Sexualidad Femenina y aborto". En: Carla Lonzi: Escupamos sobre Hegel. Y otros escritos de Liberación femenina. Buenos Aires: La Pleyade, pp. 57-66. . (1978): "Il lesbismo non è un'alternativa politica". En: Marta Lonzi, Anna Jaquinta, Moderata Fonte y Carla Lonzi: La presenza dell'uomo nel femminismo. Milán: Scritti di Rivolta Femminile, no. 9, pp. 176-177.

- Wittig, Monique (2010): El pensamiento heterosexual y otros ensayos. Madrid: Egales. 